\title{
Sporosalibacterium faouarense gen. nov., sp. nov., a moderately halophilic bacterium isolated from oil-contaminated soil
}

\author{
Correspondence \\ Marc Labat \\ marc.labat@esil.univmed.fr
}

\author{
Raja Rezgui, ${ }^{1,2}$ Zouhaier Ben Ali Gam, ${ }^{1}$ Said Ben Hamed, ${ }^{1,2}$ \\ Marie-Laure Fardeau, ${ }^{1}$ Jean-Luc Cayol, ${ }^{1}$ Abderrazak Maaroufi ${ }^{2}$ \\ and Marc Labat ${ }^{1}$
}

\author{
${ }^{1}$ Microbiologie et Biotechnologie des Environnements Chauds, UMR D180, IRD, Universités de \\ Provence et de la Méditerranée, 163 avenue de Luminy, case 925, F-13288 Marseille cedex 9, France \\ ${ }^{2}$ Laboratoire de Microbiologie, Groupe des Bioprocédés, Institut Pasteur de Tunis, BP 74, Place \\ Pasteur, Belvédère, Tunis 1002, Tunisia
}

A novel strictly anaerobic, moderately halophilic and mesophilic bacterium, designated strain SOL $3 f 37^{\top}$, was isolated from a hydrocarbon-polluted soil surrounding a deep petroleum environment located in south Tunisia. Cells of strain SOL3f37 $7^{\top}$ stained Gram-positive and were motile, straight and spore-forming. Strain SOL3f3 $7^{\top}$ had a typical Gram-positive-type cell-wall structure, unlike the thick, multilayered cell wall of its closest relative Clostridiisalibacter paucivorans. The major fatty acids were iso- $\mathrm{C}_{15: 0}(41 \%)$, iso- $\mathrm{C}_{14: 0} 3-\mathrm{OH}$ and/or iso- $\mathrm{C}_{15: 0}$ dimethyl acetal $(21.6 \%)$, iso- $\mathrm{C}_{13: 0}(4.4 \%)$, anteiso- $\mathrm{C}_{15: 0}(3.9 \%)$ and iso- $\mathrm{C}_{15: 1}(2.8 \%)$. Strain SOL3f3 $7^{\top}$ grew between 20 and $48{ }^{\circ} \mathrm{C}$ (optimum $40{ }^{\circ} \mathrm{C}$ ) and at $\mathrm{pH} 6.2-8.1$ (optimum pH 6.9). Strain SOL3f37 ${ }^{\top}$ required at least $0.5 \mathrm{~g} \mathrm{NaCl} \mathrm{I}^{-1}$ and grew in the presence of $\mathrm{NaCl}$ concentrations up to $150 \mathrm{~g} \mathrm{I}^{-1}$ (optimum $40 \mathrm{~g} \mathrm{I}^{-1}$ ). Yeast extract $\left(2 \mathrm{~g} \mathrm{I}^{-1}\right.$ ) was required for degradation of pyruvate, fumarate, fructose, glucose and mannitol. Also, strain SOL3f37 ${ }^{\top}$ grew heterotrophically on yeast extract, peptone and bio-Trypticase, but was unable to grow on Casamino acids. Sulfate, thiosulfate, sulfite, elemental sulfur, fumarate, nitrate and nitrite were not reduced. The DNA G +C content was 30.7 mol\%. Phylogenetic analysis based on 16S rRNA gene sequences revealed that strain $\mathrm{SOL} 3 \mathrm{f} 37^{\top}$ was a member of the family Clostridiaceae in the order Clostridiales; strain SOL $3 f 37^{\top}$ was related to members of various genera of the family Clostridiaceae. It exhibited highest 16S rRNA gene sequence similarity (93.4\%) with

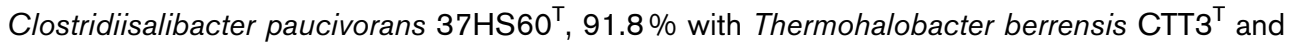
$91.7 \%$ with Caloranaerobacter azorensis $\mathrm{MV} 1087^{\top}$. On the basis of genotypic, phenotypic and phylogenetic data, it is suggested that strain SOL3f3 $7^{\top}$ represents a novel species in a new genus. The name Sporosalibacterium faouarense gen. nov., sp. nov. is proposed, with SOL3f37 ${ }^{\top}$ $\left(=\mathrm{DSM} 21485^{\mathrm{T}}=\mathrm{JCM} 15487^{\mathrm{T}}\right)$ as the type strain of Sporosalibacterium faouarense.
The Clostridiales constitutes one of the largest of the eubacterial orders. It encompasses a complex range of bacteria that may be Gram-negative or Gram-positive, psychrophilic, mesophilic or thermophilic, spore-forming or non-spore-forming, chemo-organoheterotrophic or chemolithotrophic and have been found in a variety of habitats, and it includes a large number of eukaryotic pathogens (Cato et al., 1986). The family Clostridiaceae was the first of the 19 families within the order Clostridiales to be described, and it contains the type

Abbreviation: DMA, dimethyl acetal.

The GenBank/EMBL/DDBJ accession number for the 16S rRNA gene sequence of strain SOL3f37 ${ }^{\top}$ is EU567322. genus of the order. It contains 13 recognized genera, of which the type genus, Clostridium, contains about 190 recognized species at the time of writing. Recently, the systematics of the Clostridiaceae have been found to differ significantly from previous descriptions (Wiegel, 2009). Several of the species that were not in the radiation of the genus Clostridium sensu stricto have been transferred to new genera and other families, in some cases even in different orders. The taxa presently placed into the Clostridiaceae are generally obligately anaerobic rods. Although most of the species are neutrophiles, several alkaliphilic, alkalithermophilic, moderately halophilic, haloalkaliphilic and slightly acidophilic species have been described (Wiegel, 2009). 
Here, the isolation and characterization are reported of a novel strictly anaerobic, moderately halophilic, heterotrophic, spore-forming bacterium, belonging to the family Clostridiaceae, which originated from a hydrocarbonpolluted soil surrounding a deep petroleum environment located in the El Faouar area in south Tunisia. It is suggested that this strain, SOL $3 \mathrm{f} 37^{\mathrm{T}}$, represents a novel species in a new genus of the order Clostridiales.

Samples were collected in sterile tubes from a hydrocarbonpolluted soil surrounding a deep petroleum environment located in the El Faouar area in south Tunisia, transported to our laboratory and stored at $4{ }^{\circ} \mathrm{C}$ until analysis. The $\mathrm{NaCl}$ concentration of the samples was $36 \mathrm{gl}^{-1}$. The temperature of the soil at the sampling site was $35-39{ }^{\circ} \mathrm{C}$ during the day. Samples were collected at the surface of the soil $(0-2 \mathrm{~cm}$ deep) and the $\mathrm{pH}$ of the soil was 6.8.

Enrichment and isolation were performed using an anaerobic enrichment medium, which contained (per litre distilled water) $0.3 \mathrm{~g} \mathrm{KH}_{2} \mathrm{PO}_{4}, 0.3 \mathrm{~g} \mathrm{~K}_{2} \mathrm{HPO}_{4}, 1.0 \mathrm{~g} \mathrm{NH}_{4} \mathrm{Cl}$, $40 \mathrm{~g} \mathrm{NaCl}, 0.5 \mathrm{~g} \mathrm{KCl}, 0.2 \mathrm{~g} \mathrm{CaCl}_{2}, 0.5 \mathrm{~g}$ cysteine hydrochloride, $2 \mathrm{~g}$ yeast extract (Difco), $5 \mathrm{~g}$ bio-Trypticase, $3 \mathrm{~g}$ glucose, $1 \mathrm{ml}$ mineral element solution (Widdel \& Pfennig, $1981)$ and $1 \mathrm{ml} 0.1 \%(\mathrm{w} / \mathrm{v})$ resazurin. The medium was adjusted to $\mathrm{pH} 7.1$ with $10 \mathrm{M} \mathrm{KOH}$. This enrichment medium was boiled under a stream of $\mathrm{O}_{2}$-free $\mathrm{N}_{2}$ gas and cooled to room temperature and $5 \mathrm{ml}$ aliquots were then distributed into Hungate tubes under a stream of $\mathrm{O}_{2}$-free $\mathrm{N}_{2}$ gas. The $\mathrm{N}_{2}$ gas phase was replaced with $\mathrm{N}_{2} / \mathrm{CO}_{2}$ $(80: 20)$ and the tubes were autoclaved. To initiate enrichment cultures, a small portion of the soil sample was inoculated into the growth medium and incubated at $37{ }^{\circ} \mathrm{C}$ without agitation. The Hungate technique was then used throughout isolation and study for physiological and metabolic characterization.

Enrichment cultures showed growth after $24 \mathrm{~h}$ incubation at $37{ }^{\circ} \mathrm{C}$ and microscopic examination revealed the presence of motile rod-shaped bacteria. Growth was determined by inserting culture tubes directly into a model Cary 50 Scan spectrophotometer (Varian) and measuring the $\mathrm{OD}_{580}$. Cultures were purified by repeating the Hungate roll-tube method (Hungate, 1969) and using enrichment medium solidified with $2 \%(\mathrm{w} / \mathrm{v})$ agar (Difco). Several colonies were picked and cultured in the corresponding culture medium. The isolation process was repeated several times until isolates were deemed to be axenic. Several axenic cultures were obtained using this process and one strain, named SOL $3 \mathrm{f} 37^{\mathrm{T}}$, was used for further characterization.

Cells of strain SOL $3 \mathrm{f} 37^{\mathrm{T}}$ were straight, thin and long rods $(0.5 \times 5.0-10.0 \mu \mathrm{m})$ that grew singly or in pairs. Cells were motile by means of a monotrichous laterally inserted flagellum (Fig. 1). Strain SOL $3 \mathrm{f} 37^{\mathrm{T}}$ stained Gram-positive. Spherical and terminal spores were observed, mainly in old cultures. Strain SOL $3 \mathrm{f} 37^{\mathrm{T}}$ showed a typical Gram-positivetype cell-wall structure, unlike the thick, multilayered cell wall of its closest relative Clostridiisalibacter paucivorans.

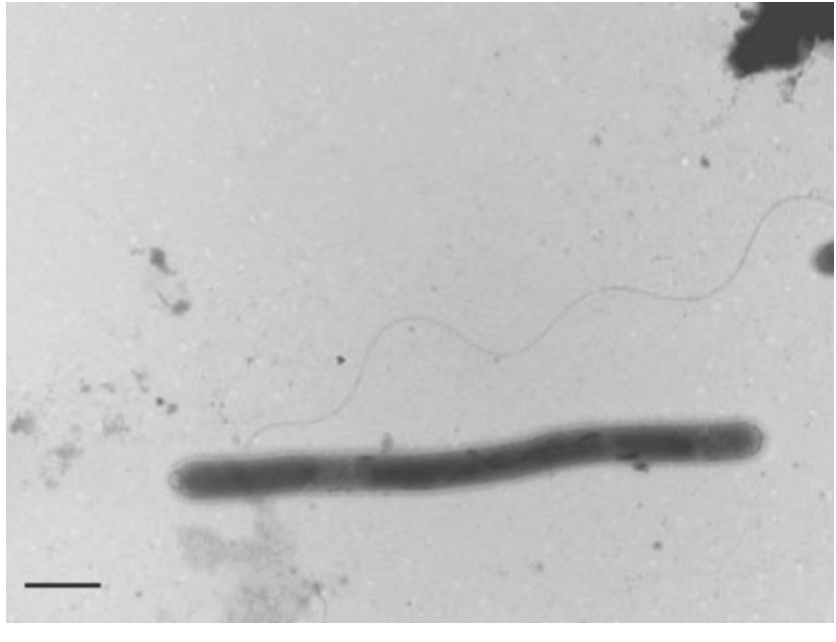

Fig. 1. Electron micrograph of a negatively stained cell of strain SOL $3 f 37^{\top}$ showing a monotrichous laterally inserted flagellum. Bar, $1 \mu \mathrm{m}$.

Fatty acid methyl esters were extracted from fresh biomass by the CCUG using the standard Microbial Identification System procedure (Microbial ID). The Microbial ID system was used to compare the fatty acid methyl esters of strain SOL $3 \mathrm{f} 37^{\mathrm{T}}$ with fatty acid patterns stored in the fatty acid database. Six fatty acid components were found at levels greater than $2 \%$ (Table 1 ), with an exceptionally high percentage of the fatty acid component iso- $\mathrm{C}_{15: 0}(41 \%)$. The other major components were identified as iso- $\mathrm{C}_{14: 0}$ 3-OH and/or iso- $\mathrm{C}_{15: 0}$ dimethyl acetal (DMA) (21.6\%), iso- $\mathrm{C}_{13: 0}(4.4 \%)$, anteiso- $\mathrm{C}_{15: 0}(3.9 \%)$, iso- $\mathrm{C}_{15: 1}(2.8 \%)$ and one major component that remained unidentified $(12.5 \%)$.

Strain SOL3f37 ${ }^{\mathrm{T}}$ did not grow in anaerobic enrichment medium containing traces of oxygen (as indicated by the pink-coloured resazurin in the growth medium) and was therefore described as a strict anaerobe. It grew anaerobically at $20-48{ }^{\circ} \mathrm{C}$, with an optimum at $40{ }^{\circ} \mathrm{C}$.

Substrate utilization was studied using anaerobic basal medium containing (per litre distilled water) $0.3 \mathrm{~g}$ $\mathrm{KH}_{2} \mathrm{PO}_{4}, 0.3 \mathrm{~g} \mathrm{~K}_{2} \mathrm{HPO}_{4}, 1.0 \mathrm{~g} \mathrm{NH}_{4} \mathrm{Cl}, 40.0 \mathrm{~g} \mathrm{NaCl}, 0.5 \mathrm{~g}$ $\mathrm{KCl}, 0.2 \mathrm{~g} \mathrm{CaCl}_{2}$ and $0.5 \mathrm{~g}$ cysteine hydrochloride. Yeast extract $\left(0.2 \mathrm{~g}\right.$ or $\left.2.0 \mathrm{~g} \mathrm{l}^{-1}\right)$ was added to this basal medium. For determination of $\mathrm{NaCl}$ requirements, $\mathrm{NaCl}$ was weighed directly into tubes at concentrations up to $200 \mathrm{~g}$ $1^{-1}$ before dispensing basal medium without $\mathrm{NaCl}$. The strain did not grow when $\mathrm{NaCl}$ was omitted from the basal medium. It grew in $\mathrm{NaCl}$ concentrations ranging from 0.5 to $150 \mathrm{~g} \mathrm{l}^{-1}$ (optimum $40 \mathrm{~g} \mathrm{l}^{-1}$ ). For $\mathrm{pH}$ studies, the same medium was adjusted to the desired $\mathrm{pH}$ using anaerobically prepared stock solutions of $\mathrm{NaHCO}_{3}(10 \%)$ or $\mathrm{Na}_{2} \mathrm{CO}_{3}$ (8\%). Growth occurred between $\mathrm{pH} 6.2$ and 8.1; the optimum $\mathrm{pH}$ was 6.9. Yeast extract $\left(2 \mathrm{~g} \mathrm{l}^{-1}\right)$ was required for degradation of the carbon substrates pyruvate, 
Table 1. Cellular fatty acid contents of strain SOL $3 f 37^{\top}$ and its closest relative, Clostridiisalibacter paucivorans $37 \mathrm{HS} 60^{\top}$

Strains: 1, Sporosalibacterium faouarense gen. nov., sp. nov. SOL3f37 ${ }^{\mathrm{T}}$; 2, Clostridiisalibacter paucivorans $37 \mathrm{HS}^{\mathrm{T}} \mathrm{T}^{\mathrm{T}}$ (data from Liebgott et al., 2008). Values are percentages (w/v) of total fatty acids. FAME, fatty acid methyl ester; DMA, dimethyl acetal; $c$, cis; cyc, cyclopropyl fatty acid; ND, no detected.

\begin{tabular}{|c|c|c|}
\hline Fatty acid & 1 & 2 \\
\hline iso- $\mathrm{C}_{11: 0}$ FAME & 0.3 & ND \\
\hline iso- $\mathrm{C}_{13: 0}$ FAME & 4.4 & ND \\
\hline anteiso- $\mathrm{C}_{13: 0}$ FAME & 0.4 & ND \\
\hline iso- $\mathrm{C}_{13: 0} 2-\mathrm{OH}$ FAME & ND & 3.2 \\
\hline iso- $\mathrm{C}_{13: 0} 3-\mathrm{OH}$ FAME and/or iso- $\mathrm{C}_{15: 1} \mathrm{I} / \mathrm{H}$ FAME & 0.9 & 1.3 \\
\hline $\mathrm{C}_{14: 0}$ FAME & 0.9 & 14.3 \\
\hline iso- $\mathrm{C}_{14: 0} 2-\mathrm{OH}$ FAME & 1.5 & ND \\
\hline iso- $\mathrm{C}_{14: 0} 3-\mathrm{OH} \mathrm{FAME}$ and/or iso- $\mathrm{C}_{15: 0} \mathrm{DMA}$ & 21.6 & ND \\
\hline iso- $\mathrm{C}_{14: 1}$ FAME & 0.6 & ND \\
\hline iso- $\mathrm{C}_{15: 1} \omega 7 c$ FAME & $\mathrm{ND}$ & 2.2 \\
\hline iso- $\mathrm{C}_{15: 1}$ F FAME & 2.8 & ND \\
\hline iso- $\mathrm{C}_{15: 0}$ FAME & 41.0 & 6.6 \\
\hline anteiso- $\mathrm{C}_{15: 0}$ FAME & 3.9 & 1.5 \\
\hline $\mathrm{C}_{15: 1} \omega 5 c$ FAME & 0.8 & ND \\
\hline $\mathrm{C}_{16: 0}$ FAME & 1.2 & 7.6 \\
\hline $\mathrm{C}_{16: 1} \omega 7 c$ alcohol & 0.3 & ND \\
\hline iso- $\mathrm{C}_{16: 1} \mathrm{I}$ and/or $\mathrm{C}_{14: 0} 3-\mathrm{OH}$ FAME & 0.3 & ND \\
\hline $\mathrm{C}_{16: 1} \omega 9 c$ FAME & $\mathrm{ND}$ & 2.5 \\
\hline $\mathrm{C}_{16: 1} \omega 7 c$ FAME & $\mathrm{ND}$ & 9.9 \\
\hline $\mathrm{C}_{16: 1} \omega 9 c$ DMA & ND & 1.8 \\
\hline $\mathrm{C}_{16: 1} \omega 7 c$ DMA & $\mathrm{ND}$ & 8.5 \\
\hline iso- $\mathrm{C}_{17: 0}$ FAME & 0.4 & ND \\
\hline $\mathrm{C}_{17: 0}$ cyc FAME & 0.6 & 2.2 \\
\hline $\mathrm{C}_{17: 0}$ cyc DMA & $\mathrm{ND}$ & 1.6 \\
\hline anteiso- $\mathrm{C}_{17: 1} \omega 9 c$ FAME & $\mathrm{ND}$ & 2.2 \\
\hline anteiso- $\mathrm{C}_{17: 1}$ B FAME and/or iso- $\mathrm{C}_{16: 1}$ & 1.5 & $\mathrm{ND}$ \\
\hline $\mathrm{C}_{17: 1} \omega 10 c$ and/or anteiso- $\mathrm{C}_{17: 1} \omega 3 c$ FAME & ND & 19.3 \\
\hline $\mathrm{C}_{18: 0}$ FAME & 1.3 & $\mathrm{ND}$ \\
\hline iso- $\mathrm{C}_{19: 1}$ FAME & 1.0 & ND \\
\hline
\end{tabular}

fumarate, fructose, glucose and mannitol (all at $20 \mathrm{mM}$ ). Furthermore, strain SOL $3 \mathrm{f} 37^{\mathrm{T}}$ grew heterotrophically on yeast extract, peptone and bio-Trypticase, but was unable to grow on Casamino acids. Fumarate was reduced to succinate. End products from pyruvate utilization were acetate, $\mathrm{H}_{2}$ and $\mathrm{CO}_{2}$. Strain $\mathrm{SOL} 3 \mathrm{f} 37^{\mathrm{T}}$ did not use cellobiose, cellulose, galactose, lactose, maltose, mannose, ribose, sucrose, xylose, melibiose, arabinose, glycerol, ethanol, methanol, acetate, propionate, butyrate, lactate, citrate, xylan or starch. Acetate was produced after growth on glucose, fructose and mannitol. None of the amino acids tested (at $20 \mathrm{mM}$ ), including threonine, glycine, proline, tyrosine, phenylalanine, leucine, isoleucine, cysteine, lysine, serine, valine, alanine, arginine, tryptophan, asparagine, aspartic acid, glutamine, glutamic acid, methionine and histidine, supported growth.

Sulfate, thiosulfate and elemental sulfur were added separately to the anaerobic basal medium at final concentrations of
$20 \mathrm{mM}, 20 \mathrm{mM}$ and $0.1 \%(\mathrm{w} / \mathrm{v})$, respectively, for potential use as an electron sink. Nitrate and nitrite utilization tests were carried out in triplicate in butyl-capped Hungate tubes filled with $5 \mathrm{ml}$ pre-reduced medium (without resazurin and $\mathrm{Na}_{2} \mathrm{~S}$ ) distributed under a nitrogen atmosphere. $\mathrm{NaNO}_{3}$ $(10 \mathrm{mM})$ or $\mathrm{NaNO}_{2}(10 \mathrm{mM})$ was added to the tubes immediately before inoculation $(10 \%, \mathrm{v} / \mathrm{v})$. These tests were carried out under similar conditions without pre-reducing the medium and without a nitrogen atmosphere for oxygen utilization.

Sulfate $(20 \mathrm{mM})$, thiosulfate $(20 \mathrm{mM})$, elemental sulfur $(0.1 \%)$, nitrate $(20 \mathrm{mM})$ and nitrite $(2 \mathrm{mM})$ were not used as electron acceptors. The sulfur test was carried out photometrically as colloidal CuS (Fardeau et al., 1997) and nitrate/nitrite reduction was assayed using specific sticks (Quantofix; Macherey-Nagel).

The DNA G + C content, determined by the Identification Service of the DSMZ (Deutsche Sammlung von Mikroorganismen und Zellkulturen $\mathrm{GmbH}$, Braunschweig, Germany) using the method of Mesbah et al. (1989), was $30.7 \mathrm{~mol} \%$.

Genomic DNA of strain SOL3f37 ${ }^{\mathrm{T}}$ was extracted using the Wizard Genomic DNA Purification kit following the manufacturer's protocol (Promega). The universal primers Fd1 (5'-AGAGTTTGATCCTGGCTCAG-3') and Rd1 (5'AAGGAGGTGATCCAGCC- $3^{\prime}$ ) were used to amplify the 16S rRNA gene. The nucleotide sequence (1497 bases) was aligned manually using the sequence alignment editor BioEdit (Hall, 1999). Reference sequences were obtained from the Ribosomal Database Project II (Maidak et al., 2001) and GenBank (Benson et al., 1999). Pairwise evolutionary distances based on 1246 unambiguous nucleotides were computed by the method of Jukes \& Cantor (1969). The phylogenetic tree obtained by the neighbour-joining method (Saitou \& Nei, 1987) is shown in Fig. 2; its topology was supported by the maximumparsimony and maximum-likelihood algorithms (not shown).

$16 \mathrm{~S}$ rRNA gene sequence analysis of strain SOL $3 \mathrm{f} 37^{\mathrm{T}}$ revealed that it was a member of the family Clostridiaceae of the order Clostridiales, as defined in the currently proposed taxonomy in Bergey's Manual of Systematic Bacteriology (Wiegel, 2009). The three most closely related strains with validly published names were Clostridiisalibacter paucivorans $37 \mathrm{HS}^{\mathrm{T}} \mathrm{T}^{\mathrm{T}}$,

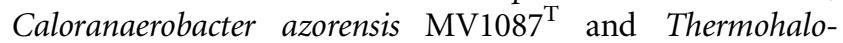
bacter berrensis $\mathrm{CTT}^{\mathrm{T}}$, with sequence similarity of 93.4 , 91.7 and $91.8 \%$, respectively. These three type strains belong to three different genera. Other closely related type strains with validly published names include Clostridium purinilyticum ATCC $33906^{\mathrm{T}}$, Clostridium acidurici ATCC $7906^{\mathrm{T}}$ and Eubacterium angustum ATCC $43737^{\mathrm{T}}$, which showed sequence similarities with strain SOL3f $37^{\mathrm{T}}$ below $92 \%$ (91.9, 91.6 and $88.8 \%$, respectively; Fig. 2).

Numerous phenotypic characteristics of strain SOL $3 \mathrm{f} 37^{\mathrm{T}}$ enabled it to be distinguished unambiguously from 


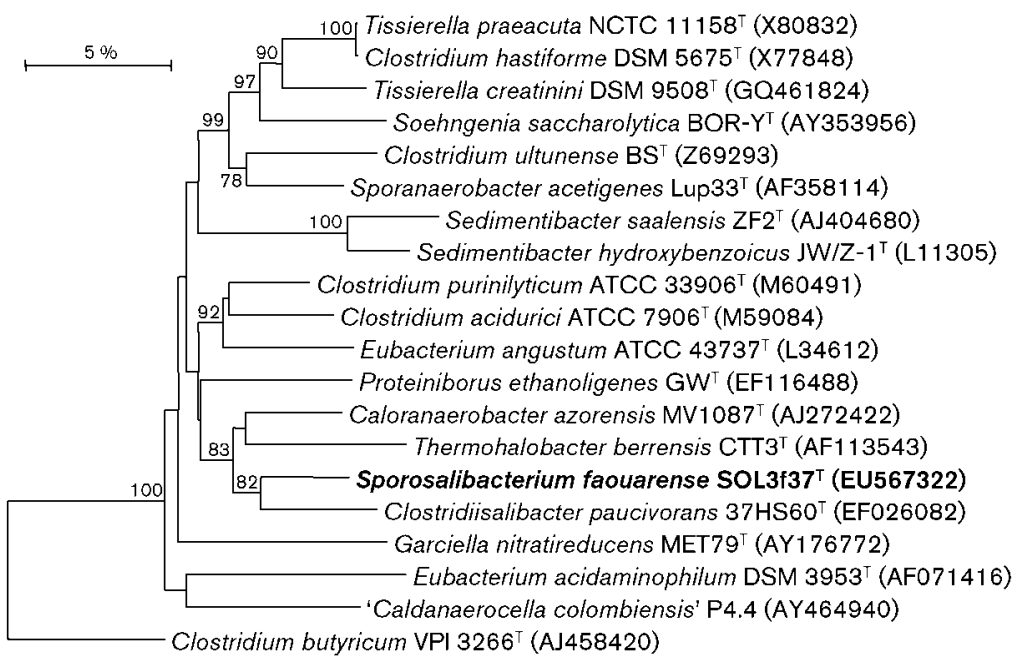

Fig. 2. Phylogenetic dendrogram based on 16S rRNA gene sequence data (1315 unambiguous alignment positions) showing the position of strain SOL $3 f 37^{\top}$. Bootstrap values (expressed as percentages of 1000 replications) greater than $70 \%$ are shown at branch points. Sequence accession numbers are given in parentheses. Bar, $5 \%$ sequence divergence.

phylogenetically closely related type strains, including Clostridium purinilyticum ATCC $33906^{\mathrm{T}}$ (Dürre et al., 1981) and Clostridium acidurici ATCC $7906^{\mathrm{T}}$ (Liebert, 1909). Isolate SOL $3 \mathrm{f} 37^{\mathrm{T}}$ was metabolically distinct from these species, which grew on purines such as uric acid and adenine and on amino acids such as glycine. However, none of them grew on sugars (Wery et al., 2001), whereas strain SOL $3 \mathrm{f} 37^{\mathrm{T}}$ grew on two sugars (glucose and fructose), but not on amino acids, including glycine.

Strain SOL $3 \mathrm{f} 37^{\mathrm{T}}$ differed markedly from Thermohalobacter berrensis $\mathrm{CTT}^{\mathrm{T}}{ }^{\mathrm{T}}$. Strain SOL $3 \mathrm{f} 37^{\mathrm{T}}$ stained Gram-positive and was spore-forming, whereas Thermohalobacter berrensis $\mathrm{CTT}^{\mathrm{T}}$ stained Gram-negative and was a non-sporulating rod (Cayol et al., 2000). Strains SOL $3 \mathrm{f} 37^{\mathrm{T}}$ and Thermohalobacter berrensis $\mathrm{CTT}^{\mathrm{T}}$ also differed in their growth conditions; strain SOL $3 \mathrm{f} 37^{\mathrm{T}}$, a slightly thermotolerant bacterium, grew between 20 and $48{ }^{\circ} \mathrm{C}$ (optimum $40{ }^{\circ} \mathrm{C}$ ), whereas Thermohalobacter berrensis $\mathrm{CTT}^{\mathrm{T}}$, a strict thermophile, grew at up to $70{ }^{\circ} \mathrm{C}$ (optimum $65{ }^{\circ} \mathrm{C}$ ), but not below $45{ }^{\circ} \mathrm{C}$.

Strain SOL $3 \mathrm{f} 37^{\mathrm{T}}$ also differed unambiguously from Caloranaerobacter azorensis MV1087 ${ }^{\mathrm{T}}$ (Wery et al., 2001). Strain SOL $3 \mathrm{f}^{\mathrm{T}}$ stained Gram-positive, whereas Caloranaerobacter azorensis $\mathrm{MV} 1087^{\mathrm{T}}$ was Gram-negative and had a characteristic Gram-negative cell wall. In addition, the DNA G $+\mathrm{C}$ content of Caloranaerobacter azorensis MV1087 ${ }^{\mathrm{T}}$ (27.0 mol\%) was significantly lower than the value calculated for strain SOL $3 \mathrm{f} 37^{\mathrm{T}}$ (30.7 mol\%). Further-

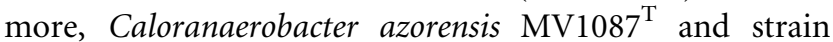
SOL $3 \mathrm{f} 37^{\mathrm{T}}$ did not use the same carbohydrates; in contrast to Caloranaerobacter azorensis $\mathrm{MV} 1087^{\mathrm{T}}$, strain SOL3f37 ${ }^{\mathrm{T}}$ was able to use glucose, fructose and mannitol but not arabinose, ribose, xylose, galactose or sorbose. Strain SOL $3 \mathrm{f} 37^{\mathrm{T}}$ also differed from Caloranaerobacter azorensis MV $1087^{\mathrm{T}}$ in terms of physiological growth conditions; the optimum growth temperature of strain SOL $3 \mathrm{f} 37^{\mathrm{T}}$ was $40{ }^{\circ} \mathrm{C}$, whereas Caloranaerobacter azorensis $\mathrm{MV}_{1087^{\mathrm{T}}}$ was able to grow at up to $70{ }^{\circ} \mathrm{C}$, with optimum growth at
$65{ }^{\circ} \mathrm{C}$. Hence, unlike Caloranaerobacter azorensis MV $1087^{\mathrm{T}}$, which is thermophilic, strain SOL $3 \mathrm{f}^{\mathrm{T}}$ was only slightly thermotolerant.

Finally, strain SOL $3 \mathrm{f} 37^{\mathrm{T}}$ could be distinguished unambiguously from Clostridiisalibacter paucivorans $37 \mathrm{HS}^{\mathrm{T}} \mathrm{T}^{\mathrm{T}}$ in terms of marked differences in their morphological, genetic and physiological traits. Clostridiisalibacter paucivorans $37 \mathrm{HS}^{\mathrm{T}}$ (Liebgott et al., 2008) was able to utilize the organic acids succinate, fumarate and pyruvate (all at $20 \mathrm{mM}$ ), whereas strain SOL $3 \mathrm{f}^{3} 7^{\mathrm{T}}$ was unable to use succinate, but could use fumarate and pyruvate, as well as glucose, fructose and mannitol (Table 2). Strain SOL $3 \mathrm{f} 37^{\mathrm{T}}$ and Clostridiisalibacter paucivorans $37 \mathrm{HS}^{\mathrm{T}} 0^{\mathrm{T}}$ both reduced fumarate slowly to succinate. End products from pyruvate fermentation were acetate, $\mathrm{H}_{2}$ and $\mathrm{CO}_{2}$. Unlike Clostridiisalibacter paucivorans $37 \mathrm{HS} 60^{\mathrm{T}}$, which was unable to use sugars as carbon sources, strain SOL $3 \mathrm{f} 37^{\mathrm{T}}$ produced acetate after growth on glucose, fructose and mannitol. Like Clostridiisalibacter paucivorans $37 \mathrm{HS}^{\mathrm{T}}{ }^{\mathrm{T}}$, strain SOL $3 \mathrm{f} 37^{\mathrm{T}}$ was an anaerobic, spore-forming, rod-shaped bacterium. However, the 16S rRNA gene sequence similarity between strain SOL $3 \mathrm{f} 37^{\mathrm{T}}$ and Clostridiisalibacter paucivorans $37 \mathrm{HS}^{\mathrm{T}} \mathrm{T}^{\mathrm{T}}$ was less than $93.4 \%$, and numerous phenotypic characteristics of isolate SOL $3 \mathrm{f} 37^{\mathrm{T}}$ were completely distinct. Strain SOL $3 \mathrm{f}^{2} 7^{\mathrm{T}}$ differed from Clostridiisalibacter paucivorans $37 \mathrm{HS}^{\mathrm{T}} 0^{\mathrm{T}}$ in its inability to use Casamino acids and amino acids. Clostridiisalibacter paucivorans $37 \mathrm{HS}^{\mathrm{T}}$ was able to use cysteine, lysine, serine and valine for growth, whereas strain SOL $3 \mathrm{f} 37^{\mathrm{T}}$ was unable to grow on any of the 20 amino acids tested. Strain SOL $3 \mathrm{f} 37^{\mathrm{T}}$ and Clostridiisalibacter paucivorans $37 \mathrm{HS}^{\mathrm{T}} \mathrm{T}^{\mathrm{T}}$ were both mesophilic and slightly thermotolerant, with a similar temperature range for growth, but Clostridiisalibacter paucivorans $37 \mathrm{HS} 60^{\mathrm{T}}$ grew at up to $50{ }^{\circ} \mathrm{C}$ (optimum $42{ }^{\circ} \mathrm{C}$ ), whereas SOL $3 \mathrm{f} 37^{\mathrm{T}}$ only grew at up to $48{ }^{\circ} \mathrm{C}$ (optimum $40{ }^{\circ} \mathrm{C}$ ). In addition, some physiological characteristics enabled SOL $3 \mathrm{f} 37^{\mathrm{T}}$ to be distinguished from its closest relative. Thus, in contrast to Clostridiisalibacter paucivorans $37 \mathrm{HS}^{\mathrm{T}}{ }^{\mathrm{T}}$, which was able to grow at $\mathrm{pH} 5.5$, strain SOL $3 \mathrm{f} 37^{\mathrm{T}}$ did not grow 
Table 2. Differential phenotypic characteristics of strain $\mathrm{SOL} 3 \mathrm{f} 37^{\top}$ and type strains of phylogenetically related species

Strains: 1, Sporosalibacterium faouarense gen. nov., sp. nov. SOL $3 \mathrm{f} 37^{\mathrm{T}}$ (data from the present study); 2, Clostridiisalibacter paucivorans $37 \mathrm{HS}^{\mathrm{T}}$ (Liebgott et al., 2008); 3, Caloranaerobacter azorensis MV1087 (Wery et al., 2001); 4, Thermohalobacter berrensis CTT3 ${ }^{\mathrm{T}}$ (Cayol et al., 2000). ND, No data available. None of the four strains was able to reduce thiosulfate or sulfate, but they all used pyruvate as a substrate.

\begin{tabular}{|c|c|c|c|c|}
\hline Characteristic & 1 & 2 & 3 & 4 \\
\hline Cell width $(\mu \mathrm{m})$ & 0.5 & 0.5 & $0.3-0.5$ & 0.5 \\
\hline Cell length $(\mu \mathrm{m})$ & $5.0-10.0$ & $3.0-8.0$ & $0.5-2.0$ & $3.0-8.0$ \\
\hline Gram type & Positive & Atypical positive & Negative & Negative \\
\hline Type of flagella* & ML & $\mathrm{P}$ & $\mathrm{P}$ & ML \\
\hline \multicolumn{5}{|l|}{ Temperature for growth $\left({ }^{\circ} \mathrm{C}\right)$} \\
\hline Range & $20-48$ & $20-50$ & $45-65$ & $45-70$ \\
\hline Optimum & 40 & 42 & 65 & 65 \\
\hline \multicolumn{5}{|l|}{$\mathrm{pH}$ for growth } \\
\hline Range & $6.2-8.1$ & $5.5-8.5$ & $5.5-9.0$ & $5.2-8.8$ \\
\hline Optimum & 6.9 & 6.8 & 7.0 & 7.0 \\
\hline \multicolumn{5}{|c|}{$\mathrm{NaCl}$ concentration for growth $\left(\mathrm{g} \mathrm{l}^{-1}\right)$} \\
\hline Range & $0.5-150$ & $10-100$ & $6.5-65$ & $20-150$ \\
\hline Optimum & 40 & 50 & 20 & 50 \\
\hline DNA G $+C$ content $(\mathrm{mol} \%)$ & 30.7 & 33.0 & 27.0 & 33.0 \\
\hline Growth on Casamino acids & - & + & + & - \\
\hline $\mathrm{S}^{0}$ reduction & - & - & + & - \\
\hline \multicolumn{5}{|l|}{ Utilization of: } \\
\hline Arginine & - & - & + & - \\
\hline Cysteine & - & + & $\mathrm{ND}$ & - \\
\hline Serine & - & + & $\mathrm{ND}$ & - \\
\hline Lysine & - & + & $\mathrm{ND}$ & - \\
\hline Valine & - & + & $\mathrm{ND}$ & - \\
\hline Fructose & + & - & + & + \\
\hline Galactose & - & - & + & - \\
\hline Glucose & + & - & + & + \\
\hline Mannose & - & - & $\mathrm{ND}$ & + \\
\hline Ribose & - & - & + & - \\
\hline Xylose & - & - & + & - \\
\hline Glycerol & - & - & $\mathrm{ND}$ & + \\
\hline Starch & - & - & + & + \\
\hline Mannitol & + & - & + & + \\
\hline Fumarate & + & + & - & - \\
\hline Succinate & - & + & - & $\mathrm{ND}$ \\
\hline
\end{tabular}

${ }^{\star}$ ML, Monotrichous, laterally inserted; P, peritrichous.

below pH 6.2. Also, unlike SOL3f37 ${ }^{\mathrm{T}}$, Clostridiisalibacter paucivorans $37 \mathrm{HS} 60^{\mathrm{T}}$ was unable to grow in media containing less than $10 \mathrm{~g} \mathrm{NaCl}^{-1}$. The maximum $\mathrm{NaCl}$ concentration for growth of Clostridiisalibacter paucivorans $37 \mathrm{HS}^{\mathrm{T}} 0^{\mathrm{T}}$ was $100 \mathrm{~g} \mathrm{l}^{-1}$, whereas strain SOL3 $337^{\mathrm{T}}$ was able to grow in $\mathrm{NaCl}$ concentrations up to $150 \mathrm{~g} \mathrm{l}^{-1}$. Also, the DNA G $+\mathrm{C}$ content of Clostridiisalibacter paucivorans $37 \mathrm{HS}^{\mathrm{T}} \mathrm{T}^{\mathrm{T}}(33.0 \mathrm{~mol} \%)$ was significantly higher than the value calculated for strain SOL $3 \mathrm{f} 37^{\mathrm{T}} \quad$ (30.7 mol\%). Clostridiisalibacter paucivorans $37 \mathrm{HS}^{\mathrm{T}}{ }^{\mathrm{T}}$ was motile by means of peritrichous flagella, whereas strain $\mathrm{SOL} 3 \mathrm{f} 37^{\mathrm{T}}$ was motile with a monotrichous laterally inserted flagellum (Fig. 1). Finally, Clostridiisalibacter paucivorans $37 \mathrm{HS} 60^{\mathrm{T}}$ showed an atypical thick Gram-positive cell-wall structure composed of dense and stratified multiple layers, which is restricted to date within these closely related genera to the genus Clostridiisalibacter, whereas strain SOL $3 \mathrm{f} 37^{\mathrm{T}}$ showed a typical Gram-positive-type cell-wall structure.

Taking into account the important differences in phylogenetic distances, strain SOL3f37 ${ }^{\mathrm{T}}$ represents a novel species in a new genus in the family Clostridiaceae in the order Clostridiales, for which the name Sporosalibacterium faouarense gen. nov., sp. nov. is proposed. This proposal is strongly supported by significant differences in genetic, metabolic and physiological traits and cell-wall structure. 


\section{Description of Sporosalibacterium gen. nov.}

Sporosalibacterium (Spo.ro.sa.li.bac.te' ri.um. Gr. n. spora a seed and, in bacteriology, a spore; L. n. sal, salis salt; L. neut. n. bacterium a rod; N.L. neut. n. Sporosalibacterium a moderately halophilic sporulated rod).

Rod-shaped, motile, Gram-positive bacteria, forming ovoid to spherical and subterminal to terminal spores. Spores appear mainly in old cultures. Exhibit a typical Gram-positive-type cell-wall ultrastructure, unlike the thick, multilayered cell wall of the closest relative Clostridiisalibacter paucivorans. Moderately halophilic and slightly thermotolerant. Strictly anaerobic, chemoorganotrophic. Able to grow on yeast extract, peptone, bio-Trypticase, pyruvate, fumarate, glucose, fructose and mannitol, but unable to grow on Casamino acids. Yeast extract is required for growth. Sulfur, sulfate, thiosulfate, nitrate and nitrite are not necessary for growth. $16 \mathrm{~S}$ rRNA gene sequence comparisons place Sporosalibacterium in the lineage of the low-G+C-content Gram-positive bacteria, in the family Clostridiaceae of the order Clostridiales. The type species is Sporosalibacterium faouarense.

\section{Description of Sporosalibacterium faouarense sp. nov.}

Sporosalibacterium faouarense (fa.ou.a.ren'se. N.L. neut. adj. faouarense from the El Faouar area in south Tunisia, where the type strain was isolated).

Displays the following properties in addition to those described for the genus. Cells are monotrichously flagellated, $0.5 \times 5.0-10.0 \mu \mathrm{m}$, occurring singly or in pairs. The major fatty acids detected are iso- $\mathrm{C}_{15: 0}$, iso- $\mathrm{C}_{14: 0} 3-\mathrm{OH}$ and/or iso- $\mathrm{C}_{15: 0}$ DMA, iso- $\mathrm{C}_{13: 0}$, anteiso- $\mathrm{C}_{15: 0}$ and iso$\mathrm{C}_{15: 1}$ and one major component that remains unidentified, with small proportions of various other fatty acids. Growth occurs at $20-48{ }^{\circ} \mathrm{C}$ (optimum $40{ }^{\circ} \mathrm{C}$ ). The optimum $\mathrm{pH}$ for growth is 6.9; growth occurs at $\mathrm{pH}$ 6.2-8.1. Optimum $\mathrm{NaCl}$ concentration for growth is $40 \mathrm{~g} \mathrm{l}^{-1}$; grows in the presence of $\mathrm{NaCl}$ concentrations up to $150 \mathrm{~g} \mathrm{l}^{-1}$. Heterotrophic. Requires yeast extract in order to degrade pyruvate, fumarate, fructose, glucose and mannitol. Does not use elemental sulfur, sulfate, thiosulfate, sulfite, nitrate, nitrite or oxygen as electron acceptors.

The type strain is SOL $3 \mathrm{f} 37^{\mathrm{T}}\left(=\mathrm{DSM} 21485^{\mathrm{T}}=\mathrm{JCM}\right.$ $15487^{\mathrm{T}}$ ), isolated from a hydrocarbon-polluted soil surrounding a deep petroleum environment located in the El Faouar area in south Tunisia. The G $+\mathrm{C}$ content of DNA of the type strain is $30.7 \mathrm{~mol} \%$ (HPLC).

\section{Acknowledgements}

We gratefully acknowledge Olfa Houari for valuable advice and Pierre-Pol Liebgott for technical assistance. Many thanks to Pierre Thomas and Manon Joseph for electronic microscopy and Faten Trakhna for assistance with drafting.

\section{References}

Benson, D. A., Boguski, M. S., Lipman, D. J., Ostell, J., Ouellette, B. F. F., Rapp, B. A. \& Wheeler, D. L. (1999). GenBank. Nucleic Acids Res 27, 12-17.

Cato, E. P., George, W. L. \& Finegold, S. M. (1986). Genus Clostridium Prazmowski 1880, 23 ${ }^{\mathrm{AL}}$. In Bergey's Manual of Systematic Bacteriology, vol. 2, pp. 1141-1200. Edited by P. H. A. Sneath, N. S. Mair, M. E. Sharpe \& J. G. Holt. Baltimore: Williams \& Wilkins.

Cayol, J. L., Ducerf, S., Patel, B. K. C., Garcia, J. L., Thomas, P. \& Ollivier, B. (2000). Thermohalobacter berrensis gen. nov., sp. nov., a thermophilic, strictly halophilic bacterium from a solar saltern. Int $J$ Syst Evol Microbiol 50, 559-564.

Dürre, P., Andersch, W. \& Andreesen, J. R. (1981). Isolation and characterization of an adenine-utilizing, anaerobic sporeformer, Clostridium purinolyticum sp. nov. Int J Syst Bacteriol 31, 184-194.

Fardeau, M.-L., Ollivier, B., Patel, B. K. C., Magot, M., Thomas, P., Rimbault, A., Rocchiccioli, F. \& Garcia, J.-L. (1997). Thermotoga hypogea sp. nov., a xylanolytic, thermophilic bacterium from an oilproducing well. Int J Syst Bacteriol 47, 1013-1019.

Hall, T. A. (1999). BioEdit: a user-friendly biological sequence alignment editor and analysis program for Windows 95/98/NT. Nucleic Acids Symp Ser 41, 95-98.

Hungate, R. E. (1969). A roll tube method for cultivation of strict anaerobes. Methods Microbiol 3B, 117-132.

Jukes, T. H. \& Cantor, C. R. (1969). Evolution of protein molecules. In Mammalian Protein Metabolism, vol. 3, pp. 21-132. Edited by H. N. Munro. New York: Academic Press.

Liebert, F. (1909). The decomposition of uric acid by bacteria. Proc Acad Sci Amsterdam 12, 54-64.

Liebgott, P. P., Joseph, M., Fardeau, M. L., Cayol, J. L., Falsen, E., Chamkh, F., Qatibi, A. I. \& Labat, M. (2008). Clostridiisalibacter paucivorans gen. nov., sp. nov., a novel moderately halophilic bacterium isolated from olive mill wastewater. Int $J$ Syst Evol Microbiol 58, 61-67.

Maidak, B. L., Cole, J. R., Lilburn, T. G., Parker, C. T., Jr, Saxman, P. R., Farris, R. J., Garrity, G. M., Olsen, G. J., Schimdt, T. M. \& Tiedje, J. M. (2001). The RDP-II (Ribosomal Database Project). Nucleic Acids Res 29, 173-174.

Mesbah, M., Premachandran, U. \& Whitman, W. B. (1989). Precise measurement of the $\mathrm{G}+\mathrm{C}$ content of deoxyribonucleic acid by highperformance liquid chromatography. Int J Syst Bacteriol 39, 159-167.

Saitou, N. \& Nei, M. (1987). The neighbor-joining method: a new method for reconstructing phylogenetic trees. Mol Biol Evol 4, 406425.

Wery, N., Moricet, J. M., Cueff, V., Jean, J., Pignet, P., Lesongeur, F., Cambon-Bonavita, M. A. \& Barbier, G. (2001). Caloranaerobacter azorensis gen. nov., sp. nov., an anaerobic thermophilic bacterium isolated from a deep-sea hydrothermal vent. Int J Syst Evol Microbiol 51, 1789-1796.

Widdel, F. \& Pfennig, N. (1981). Studies on dissimilatory sulfatereducing bacteria that decompose fatty acids. I. Isolation of new sulfate-reducing bacteria enriched with acetate from saline environments. Description of Desulfobacter postgatei gen. nov., sp. nov. Arch Microbiol 129, 395-400.

Wiegel, J. (2009). Family I. Clostridiaceae Pribram 1933, 90 ${ }^{\mathrm{AL}}$. In Bergey's Manual of Systematic Bacteriology, 2nd edn, vol. 3, pp. 736738. Edited by P. De Vos, G. M. Garrity, D. Jones, N. R. Krieg, W. Ludwig, F. A. Rainey, K.-H. Schleifer \& W. B. Whitman. New York: Springer. 